\title{
Effect of spatial resolution on information content characterization in remote sensing imagery based on classification accuracy
}

\author{
R. M. NARAYANAN*, M. K. DESETTY \\ Department of Electrical Engineering and Center for Electro-Optics, University \\ of Nebraska, Lincoln, NE 68588-0511, USA \\ and S. E. REICHENBACH \\ Department of Computer Science and Engineering, University of \\ Nebraska, Lincoln, NE 68588-0115, USA
}

(Received 30 August 1999; in final form 28 August 2000)

\begin{abstract}
The information content of remote sensing imagery depends upon various factors such as spatial and radiometric resolutions, spatial scale of the features to be imaged, radiometric contrast between different target types, and also the final application for which the imagery has been acquired. Various textural measures are used to characterize the image information content, based upon which different image processing algorithms are employed to enhance this quantity. Previous work in this area has resulted in three different approaches for quantifying image information content, primarily based on interpretability, mutual information, and entropy. These approaches, although well refined, are difficult to apply to all types of remote sensing imagery. Our approach to quantifying image information content is based upon classification accuracy. We propose an exponential model for information content based upon target-background contrast, and target size relative to pixel size. The model is seen to be applicable for relating information content to spatial resolution for real Landsat Thematic Mapper (TM) as well as Shuttle Imaging Radar-C (SIR-C) images. An interesting conclusion that emerges from this model is that although the TM image has higher information content than the SIR-C image at smaller pixel sizes, the opposite is true at larger pixel sizes. The transition occurs at a pixel size of about $720 \mathrm{~m}$. This tells us that for applications that require high resolution (or smaller pixel sizes), the TM sensor is more useful for terrain classification, while for applications involving lower resolutions (or larger pixel sizes), the SIR-C sensor has an advantage. Thus, the model is useful in comparing different sensor types for different applications.
\end{abstract}

\section{Introduction}

Remote sensing images are formed by recording the reflected energy or radiance from a target scene. In remote sensing terminology, an image refers to a twodimensional representation of the energy reflected from or emitted by the scene. Modern remote sensing sensors use digital systems to store and process the image

*e-mail: rnarayanan@unl.edu 


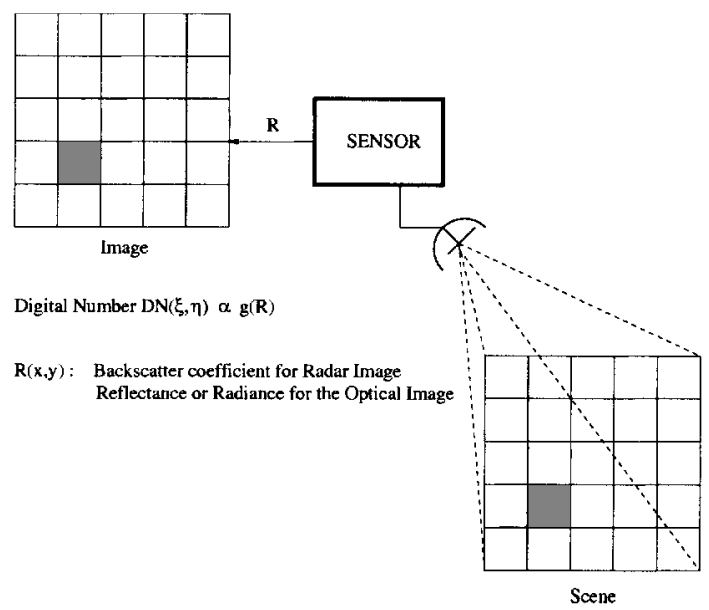

Figure 1. Scene to image transformation.

data. A typical digital image shown in figure 1 consists of a two-dimensional array of pixels, each with a grey level, $n$. The pixel coordinates are labelled $(\xi, \eta)$ on the image for the coordinates $(x, y)$ on the ground cell. If $n_{\mathrm{b}}$ represents the number of bits to represent the digital image, then $n$ may take an integer value between 0 and $\left(2^{n_{\mathrm{b}}}-1\right)$.

Each pixel in the image represents the average reflectance or emittance of the target on the ground: darker areas representing lower values, while brighter areas represent higher values. The brightness of each pixel depends on the size of the scatterers within the pixel, properties of targets contained within the pixel, the wave polarization, and the viewing angle, and the target's chemical composition. Brightness also depends on surface roughness relative to wavelength.

Remote sensing images acquired in various spectral bands are used to estimate certain geophysical parameters or detect the presence or extent of geophysical phenomena. Examples include the estimation of soil moisture or delineation of the ice-water boundary in polar regions using synthetic aperture radar (SAR) imagery. In a majority of cases, the raw image acquired by the sensor is processed using various operations such as filtering, compression, enhancement, and others. In all of these cases, the analyst is attempting to maximize the information content in the image to fulfil the end objective. While this appears deceptively simple, there are a variety of issues that need to be addressed in order that available information content is maximized for a particular application. Remotely sensed images have information of varying value. Different methods of sensing and processing the data are needed to extract the maximum amount of information from the image effectively. Since information of high value is not always the easiest or most effectively obtained, an analyst needs to identify categories of high information value against the kind of information sensed and identified.

The information content needs to be quantified before an attempt to perform operations to increase its value for a particular application. However, information content is not easily quantifiable. Various methodologies have been proposed to characterize the information content of remotely sensed data (Dowman and Peacegood 1989, Kalmykov et al. 1989, Oliver 1991, Blacknell and Oliver 1993), and relate it to several variables such as resolution (both radiometric as well as 
spatial), scale of variability of the geophysical parameter of interest, image statistics, etc. It is also important to recognize that the same image may contain different amounts of information depending on the application. To illustrate this point, consider a digital image of a scene containing targets and features of different sizes and extents. Although the spatial resolution of the system may be poor, it may still be useful in identifying those targets and features of interest as long as their sizes are much larger than the sensor spatial resolution. We can then say that the information content of the image for identifying targets and features is high. On the other hand, it may be impossible to identify targets and features of sizes much smaller than the sensor spatial resolution using the same image. In this case, we say that the information content of the image for identifying targets and features of interest is low. Thus, the same image contains high information content for delineating large-sized targets, but low information content for identifying small-sized targets. Choosing an appropriate and meaningful spatial resolution for a particular application is therefore an important task for the remote sensing analyst (Atkinson and Curran 1997).

The concept of quantification of information content in remote sensing images has been addressed in three different ways in the past. An experiment was performed to study the effect of spatial resolution on radar image interpretation, which postulated that the interpretability or information content can be determined by the 'spatial-grey-level (SGL) resolution volume' (Moore 1979). Later, a statistical model based on per-pixel basis was realized where an imaging radar was portrayed as a noisy communication channel with multiplicative noise, and an information-theoret ical approach was used to quantify the information content (Frost and Shanmugam 1983). Another approach to the quantification of the information content of optical sensor data from the Landsat-4 Thematic Mapper (TM) and Multi-Spectral Scanner (MSS) was made using the classical information theory developed by Shannon (Price 1984). In the later 1990s, information theoretic concepts were used to provide a mathematical framework for characterizing the information content of coherent images for both additive and multiplicative noise (Blacknell and Oliver 1993). Shannon's information theory was also used to compute the redundancy of L- and C-band Shuttle Imaging Radar-C (SIR-C) imagery, thereby improving land cover classification results (Le Hégarat-Mascle et al. 1997).

The interpretability model is based on visual ability to identify different targets at degraded resolutions. It does not use the image data in any mathematical computations to identify the targets, or in the computation of information content. It also does not account for the size of the target being considered, which is as important as spatial resolution in identification of the target. The mutual information model measures the information about the target from the image. This model is more applicable in the design of imaging systems for applications such as soil moisture estimation where the recorded data should be as close to the target reflectivity as possible to get accurate estimates of soil moisture. The entropy model evaluates information in terms of image gathering capability which are useful in efficient data compression and data transmission capabilities. Thus, in order to investigate the effect of spatial resolution and target size in the identification of the targets, a different model is proposed.

Table 1 shows typical generic applications that make use of remote sensing imagery, and lists specific examples in each generic category. One logical approach for quantification of information content may be based on classification accuracy, as classification procedures are used to extract information about the scene from the 
Table 1. Typical applications using remote sensing imagery.

\begin{tabular}{ll}
\hline Generic applications & \multicolumn{1}{c}{ Specific examples } \\
\hline Edge detection & $\begin{array}{l}\text { Ice-water edge in polar regions } \\
\text { Water-vegetation edge in wetlands } \\
\text { Vegetation types in mixed forest } \\
\text { Classification }\end{array}$ \\
Soil types in desert regions \\
Feature detection & $\begin{array}{l}\text { Landmines in inhomogeneous soil } \\
\text { Targets in cluttered background }\end{array}$ \\
Parameter estimation & Soil moisture patterns \\
& Vegetation biomass variability \\
\hline
\end{tabular}

remotely sensed data. In this paper, we propose a simple mathematical model to relate information content in images based on classification accuracy to the spatial resolution. Such a formulation enables us to understand the type of mathematical operations required on the imagery to improve the information content. The model was tested on Landsat TM optical and SIR-C radar data.

\section{Proposed information content model based on scene classification}

Classification of images is performed to delineate areas in the image possessing common features. This operation gives information about the scene in the image, rather than just the numerical data. Image classification produces a thematic map of the region where the themes include vegetation, soil, water bodies, etc. A classified image consists of labels of a particular landcover or type of soil present in the image. By labelling, the data has some informational value rather than just a set of digital numbers. Multispectral images, which may contain spectral characteristics from several bands with at least 8 bits/pixel per band, are reduced to a single band informational image with less than 8 bits/pixel. Spatial resolution of the image and scale of the target of interest are very important parameters in classification of images (Cao and Lam 1997). The spatial resolution determines the degree and type of information that can be extracted from an image. For example, TM imagery, which has a spatial resolution of $30 \mathrm{~m}$, can be used to extract the types of crops, trees, urban areas in Nebraska, whereas the Advanced Very High Resolution Radiometer (AVHRR) imagery, which has a resolution of $1 \mathrm{~km}$, cannot be used for this purpose, but in turn can be used to provide global land cover.

Numerous textural measures are used to characterize the local and global variability in a remotely sensed image. These measures include the mean, the standard deviation with respect to windows of various sizes, gradients in different directions, and correlations between textural parameters at different locations (Haralick et al. 1973, Tamura et al. 1978, Tomita and Tsuji 1990, Potopav et al. 1991, Shen and Srivastava 1996). Radar image simulations were performed at C-band frequency at an incidence angle of $30^{\circ}$ in order to understand the effect of spatial resolution and spatial extent of the object on the image information content (Narayanan et al. 1997), and the salient results are briefly described to develop the framework for subsequent sections of the paper. The local statistics of the image was used to identify targets of various spatial extents in cluttered background. The local mean (Lee 1980) was used in classifying a pixel as belonging to either the target or the background using a distance measure based on mean radar reflectances. The targets were chosen 
to represent both small as well as large differences between their mean radar reflectances and that of the clutter. Spatial resolution was degraded by convolving a local mean filter with equal weights of different window sizes for the images, and the average value of local mean for each target was computed using the averaging aggregation method (Bian and Butler 1999). As the size of the window increased, more of the background pixels were mixed with the target pixels and the mean reflectance of the target approached the background. The rate of advance depended on the size of the target under consideration: the target mean attained the background mean faster for the small-sized targets than for the large-sized targets. Typical images were generated using the above guidelines to provide a clear understanding of the effect of spatial resolution. It was found that the contrast between the target and the background had a major role in accurately classifying the target pixels. For the low contrast case, the classification accuracy was low, while for the high contrast case, the classification accuracy was high for the same spatial resolution. The information content, $I^{*}$, was calculated from the simulated images for each case using the equation

$$
I^{*}=1-\left(\frac{m^{*}-m_{\mathrm{T}}}{m_{\mathrm{B}}-m_{\mathrm{T}}}\right)
$$

where $m^{*}$ is the mean reflectance of the target area after using the local mean filter, $m_{\mathrm{T}}$ is the mean reflectance of the target, and the $m_{\mathrm{B}}$ is the mean reflectance of the appropriate background. For high spatial resolution, $m^{*} \approx m_{\mathrm{T}}$, and we have the target pixels mostly correctly classified; hence $I^{*} \approx 1$. For coarser spatial resolution, $m \approx m_{\mathrm{B}}$, hence the target pixels will mostly be classified wrong, so we obtain $I^{*} \approx 0$. The information content therefore varies between one for high spatial resolution and nearly zero for coarser spatial resolution.

As the spatial resolution improves and the pixel size, $\Delta R$, reduces, the amount of information to delineate the spatial extent of the target, $R$, increases. We obtain high information when the pixel size approaches zero, while the information content reduces to zero at pixel size of $\infty$, i.e. no information. The information content data from the simulated images was plotted versus $\Delta R / R$. It was found that it followed an exponential decline. Hence, we modelled the information content, $I$, as a function of pixel size, $\Delta R$, and the target characteristic dimension, $R$, as

$$
I=\exp \left[-k\left(\frac{\Delta R}{R}\right)^{n}\right]
$$

where $k$ and $n$ are the best-fit parameters related to the interpretability of the image, as well as the contrast between the target and the background. The above formulation is intuitively satisfying, since the information content is unity for $\Delta R=0$, and is zero for $\Delta R=\infty$. Using simulations to provide data points, various best fit values can be computed from the above equations. Rearranging and taking natural logarithm on both sides, we get

$$
\ln (1 / I)=k\left(\frac{\Delta R}{R}\right)^{n}
$$

Again, taking the natural logarithm, we have

$$
\ln (\ln (1 / I))=\ln k+n \ln \left(\frac{\Delta R}{R}\right)
$$


Equation (4) is in the form of a linear equation $Y=m X+C$, where $Y=\ln (\ln (1 / I))$ and $X=\ln (\Delta R / R)$. From this, the slope and intercept of equation (4) can be used to find the values of $k$ and $n$ for each case. The slope $m$ is equal to the parameter $n$, while the intercept $C$ is equal to $\ln k$, from which $k=\exp (C)$. Extension of the above formulation to real data from the Landsat TM and SIR-C sensors are discussed in the following sections.

\section{Site and image data description}

\subsection{Washita' '94 test site details}

The images used were a part of a large scale hydrological field experiment conducted over the Washita watershed near Chickasha, Oklahoma. During the experiment, two SIR-C missions were planned for April and August of 1994. The spring mission occurred from 11-17 April 1994 and fall mission from 2-6 October 1994. The Little Washita River watershed covers 235.6 square miles and is a tributary of the Washita River in south-west Oklahoma. The watershed is in the northern part of the Great Plains of the United States. Land use in this area can be grouped into several categories: range, pasture, crop land, oil waste land, quarries, urban/highways, and water. The actual data for the SIR-C and the TM were made available on a CD-ROM. This CD-ROM was released by the US Department of Agriculture, NASA and Princeton University to facilitate research by various investigators. A detailed description of the dataset can be found in a USDA report (US Department of Agriculture 1996) and on the CD-ROM. The SIR-C and TM data were provided in uncompressed TIFF-byte image format. All the datasets were georeferenced to the TM data.

\subsection{TM dataset description}

The Landsat TM data used were acquired by USDA ARS located in Durant, Oklahoma. The full image was georegistered by the USDA ARS Hydrology Lab to USDS topographic map and then the primary study area was extracted. The image was acquired on 12 April 1994. The processed files contain 934 lines by 1467 pixels at a resolution of $30 \mathrm{~m}$. Geolocation information is as follows: Output Georeferenced Units UTM 14 S E000; Projection Universal Transverse Mercator Zone 14S; and Earth Ellipsoid Clarke 1866 (NAD 27). Table 2 shows the image coordinates. The visible and infrared images of the complete TM dataset for the Washita watershed region acquired in April 1994 as well are shown in figure 2.

\subsection{SIR-C dataset description}

The SIR-C images were simultaneously acquired at two microwave wavelengths: L-band $(24 \mathrm{~cm})$ and C-band $(6 \mathrm{~cm})$. Vertical and horizontal polarized tran-

Table 2. Coordinates of the TM image for the Washita Watershed in UTM projection.

\begin{tabular}{lcc}
\hline Location & Longitude & Latitude \\
\hline Upper left corner & $562000 \mathrm{E}$ & $3875000 \mathrm{~N}$ \\
Upper right corner & $606010 \mathrm{E}$ & $3875000 \mathrm{~N}$ \\
Image centre & $584005 \mathrm{E}$ & $3860990 \mathrm{~N}$ \\
Lower left corner & $562000 \mathrm{E}$ & $3846980 \mathrm{~N}$ \\
Lower right corner & $606010 \mathrm{E}$ & $3846980 \mathrm{~N}$ \\
\hline
\end{tabular}




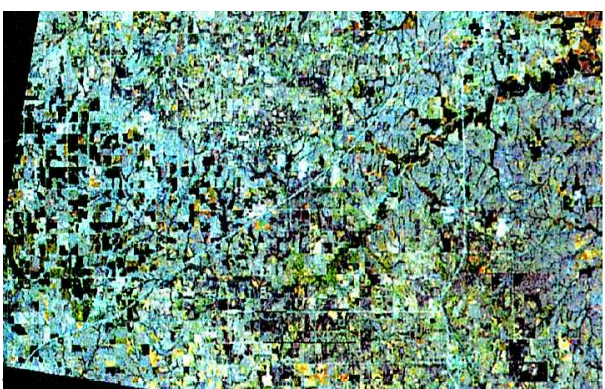

(a)

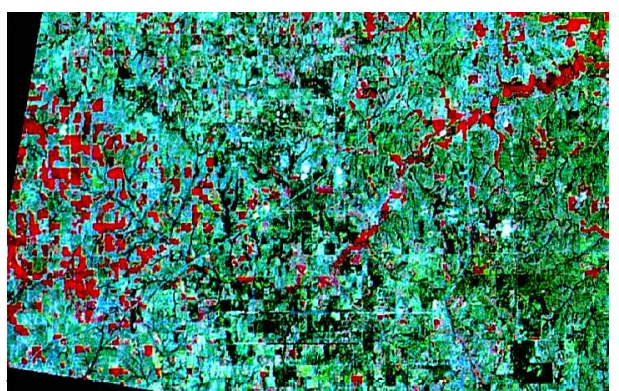

(b)

Figure 2. Visible and infrared images of the TM data. (a) True colour image using TM bands 3, 2 and 1 in RGB channels. (b) Colour infrared image using TM bands 4, 3 and 2 in RGB channels.

smitted waves were received on two separate channels, so that four polarization combinations, i.e. amplitudes and relative phase differences for the $\mathrm{HH}$ (Horizontally transmitted, Horizontally received), VV, HV and VH, are obtained. Polarimetric data provided more detailed information about the surface geometric structure, vegetation cover, and subsurface discontinuities than image brightness or intensity alone. The original SIR-C datasets processed as single look complex (slc) or multiple look complex (mlc) data and calibrated by NASA JPL were assembled by the NASA GSFC Hydrological Sciences Branch. A description of the datasets available from April 1994 are listed in table 3.

NASA JPL software was modified to output the data as a scaled backscattering coefficient. The radar backscatter coefficients were extracted from the data using the formulae:

$$
\begin{aligned}
\text { C-band HH and VV: } & d B=(D N /(255 / 25))-25 \\
\text { C-band HV: } & d B=(D N /(255 / 25))-35 \\
\text { L-band HH and VV: } & d B=(D N /(255 / 35))-35 \\
\text { L-band HV: } & d B=(D N /(255 / 35))-45
\end{aligned}
$$

These datasets were georeferenced for each day to the TM image using control points. The primary study area was extracted from the original data and consisted of 934 lines by 1467 pixels with a resolution of $30 \mathrm{~m}$.

Table 3. SIR-C data specifications during the Washita '94 experiment.

\begin{tabular}{lcccc}
\hline Date & SIR-C motion & Look angle $\left(^{\circ}\right)$ & Polarizations & Processing \\
\hline 11 April 1994 & ascending & 28.0 & HH HV VV & slc \\
12 April 1994 & ascending & 42.3 & HH HV VV & slc \\
14 April 1994 & ascending & 56.3 & HH HV & slc \\
14 April 1994 & descending & 48.3 & HH HV & slc \\
15 April 1994 & ascending & 60.2 & HH HV VV & mlc \\
15 April 1994 & descending & 42.4 & HH HV VV & slc \\
16 April 1994 & descending & 36.2 & HH HV VV & slc \\
17 April 1994 & descending & 30.9 & HH HV VV & slc \\
18 April 1994 & descending & 26.5 & HH HV VV & slc \\
\hline
\end{tabular}


The JPL software produces a scaled output designed to maximize the contrast in each band and polarization on each day individually. The output is a scaled DN of amplitude and the program outputs the scale factor. Typical JPLSCALE images are shown in figure 3. Radar backscatter was computed from the data using the following relations:

$$
\begin{aligned}
& \text { Amplitude }=(D N) / \text { ScaleFactor } \\
& \text { Power }=(\text { Amplitude })^{2} \\
& d B=10_{\log _{10}} \text { Power }
\end{aligned}
$$

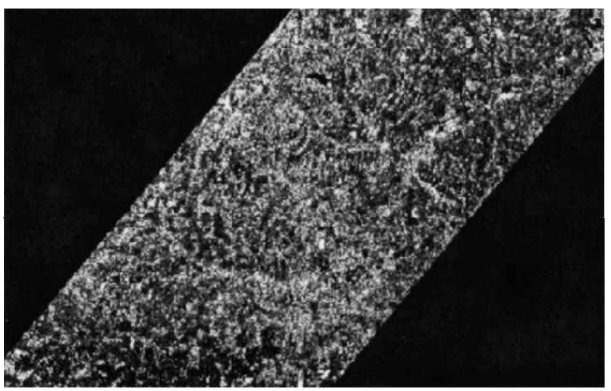

(a)

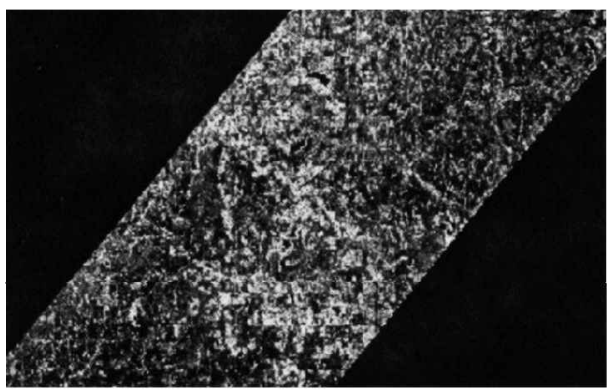

(c)

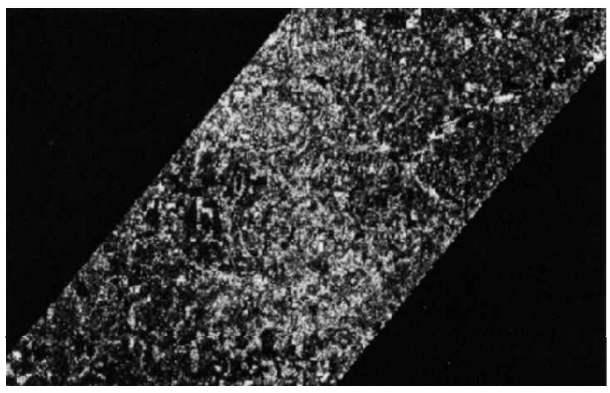

(e)

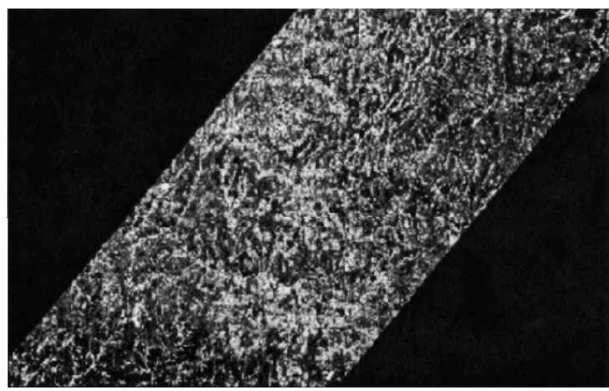

(b)

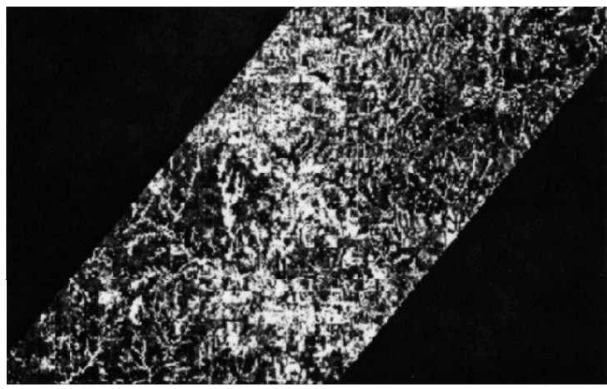

(d)

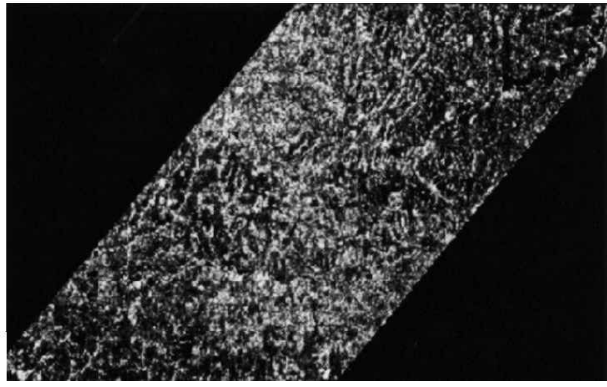

(f)

Figure 3. C- and L-band JPLSCALE SIR-C April mission images with different polarizations. The polarized SIR-C images are as follows: (a) C-band HH, $(b)$ L-band HH, (c) C-band HV, (d) L-band HV, (e) C-band VV, $(f)$ L-band VV. 


\section{Application of model to data from TM and SIR-C sensors}

\subsection{Procedure for inducing spatial degradation}

The previous investigations from the simulated data were extended to actual data acquired by two different sensors, namely Landsat TM and SIR-C. The TM sensor had greater swath, which is clearly evident from figures 2 and 3 as compared with the SIR-C data. Moreover, the SIR-C and the TM satellites had different orbital paths; hence the SIR-C image data were georeferenced and georegistered to the TM data. The area common to both the TM and SIR-C images were extracted using a mask of the SIR-C image. This mask was applied to the TM image to extract the area common to both the images. The latitude and the longitude coordinates were cross-checked for the common area. These images are shown in figures 4 and 5 . Since the SIR-C images were contaminated with speckle, an adaptive Lee filter of size $3 \times 3$ was used to remove the speckle from the images. Lee filtering is a standard deviation based (sigma) filter used to smooth speckled data whose intensity is related to the image scene, but which have an additive and/or multiplicative component (Lee 1986). It filters the data based on statistics calculated within individual filter windows. Unlike a typical low-pass smoothing filter, the Lee filter and other similar sigma filters preserve image sharpness and detail while suppressing noise. The noise model was chosen to be multiplicative of the form $z=x v$, where $z$ is the observed

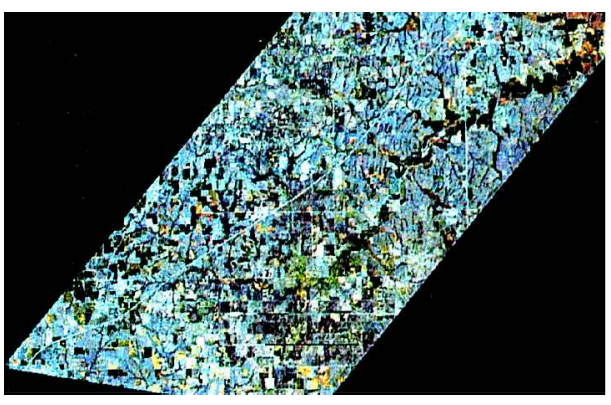

(a)

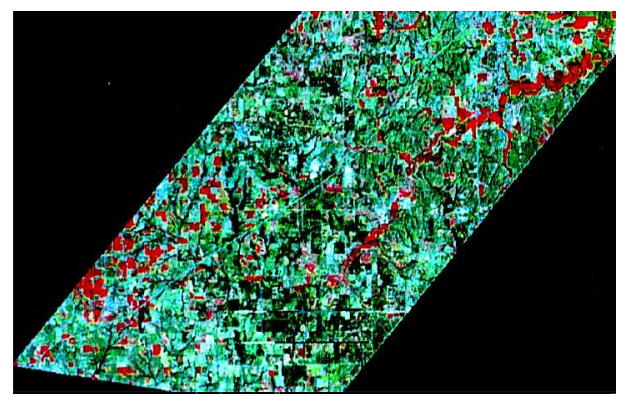

(b)

Figure 4. Visible and infrared images showing the common area after applying the mask. (a) True colour image using TM bands 3, 2 and 1 in RGB channels. (b) Infrared image using TM bands 4,3 and 2 in the RGB channels.

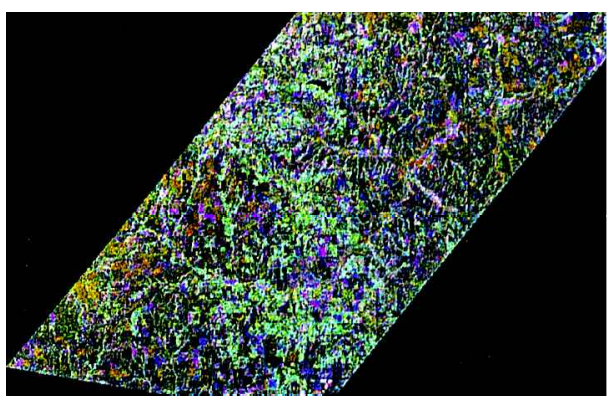

(a)

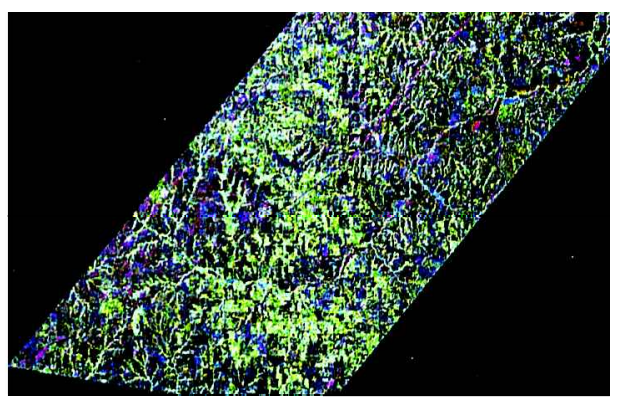

(b)

Figure 5. C- and L-band colour composite images showing the common area after applying the mask. (a) C-Band image using $\mathrm{HH}, \mathrm{HV}$ and VV in the RGB channels. (b) L-Band image using $\mathrm{HH}, \mathrm{HV}$ and $\mathrm{VV}$ in the RGB channels. 
pixel, $x$ is the noise-free image pixel, $v$ is the noise with mean 1 and standard deviation $\sigma_{v}$. In our study, a value of unity was assigned to the noise mean and variance because it is a single look image, and the noise variance can be estimated by $1 /$ (number of looks). The algorithm is given by

$$
\begin{gathered}
\hat{x}=\bar{x}+k(z-\bar{x}) \\
k=\frac{\operatorname{var}(x)}{\bar{x}^{2} \sigma_{v}^{2}+\operatorname{var}(x)}
\end{gathered}
$$

where $\hat{x}$ is the estimated pixel, $\bar{x}$ and $\operatorname{var}(x)$ are a priori mean and variance of noisefree pixel, respectively. The a priori mean and variance can be estimated by

$$
\bar{x}=\frac{\bar{z}}{\bar{v}} \quad \text { and } \quad \operatorname{var}(x)=\frac{\operatorname{var}(z)+\bar{x}^{2}}{\sigma_{v}^{2}+\bar{v}^{2}}-\bar{x}^{2}
$$

The spatial degradations were simulated by building a convolution filter with equal weights with the following sizes: $3 \times 3,5 \times 5,7 \times 7,9 \times 9,11 \times 11$ and $15 \times 15$. These filters performed spatial averaging for the entire images for all the six bands for the TM images and for the three different polarizations ( $\mathrm{HH}, \mathrm{HV}, \mathrm{VV}) \mathrm{C}$ - and L-band images for the SIR-C data.

\subsection{Information content analysis}

The analysis of the information content in real data was carried out on the basis of classification accuracy. It should be noted that this study deals with the characterization of information content using classification accuracy, but not with the comparison of classification accuracy for different sensors. The classification accuracy is a reasonable parameter to characterize the information content in an image, because a thematic map contains information about different classes in the scene. The misclassification of the pixel tells us that we are losing information about the scene. The idea to use this measure comes from our simulation results. In the simulation studies described in $\$ 2$, the information content was calculated based on a distance measure between the mean reflectance between two classes: target and background. It was inferred that when the target mean deviates towards the background mean with increasing pixel size, it was misclassified as background. The study on the simulated data was similar to the evaluation of classification accuracy at various spatial degradations. Hence this measure was used for the SIR-C and TM data which consists of many classes. Supervised classification was performed using the Maximum Likelihood Classifier (ML) (Schowengerdt 1997). Maximum Likelihood classification assumes that the statistics for each class in each band are normally distributed and calculates the probability that a given pixel belongs to a specific class. Each pixel is assigned to the class that has the highest probability (i.e. the 'maximum likelihood'). The Maximum Likelihood is based on the Bayes Decision Rule where a pixel is assigned to that class which has a posteriori probability greater than that for all other classes.

Since there were no thematic maps for the region at resolution of $30 \mathrm{~m}$, the 'ground truth' (ground data) image was obtained by classifying the region using both the optical (TM) and microwave (SIR-C) data together as a 12-band image in order to capture the maximum information about the scene. While this method cannot be used as a substitute for the actual ground data, it was felt that this approach was 
justified based upon recent results that showed considerable improvement in classification accuracy using a combination of microwave and optical data (Metternicht and Zinck 1998). The 12-band image consisted of bands 1-7, except for band 6 which is a thermal band at $120 \mathrm{~m}$ resolution, for the TM image, and three polarizations (HH, HV, VV) for both L- and C-band SIR-C images. The visible and infrared images of TM and colour composite SIR-C images were utilized to develop the training sites for the six terrain classes to be discriminated by the classifier. The six different classes identified are shown in table 4.

The different regions of interest were selected by observing the images and by analysing the NS001 images. The NS001 is a multispectral scanner operating in the Landsat spectral wavelength region aboard a NASA C-130 airplane flown over the region. The statistics of each class were calculated by taking more than one region of interest and at least 1000 data points for each class. After the statistics were calculated, the classifier was run on the whole image for both the TM and SIR-C data. First, the 'ground truth' image in figure 6 was obtained by using ML classifier using all the 12 bands of data. The statistics for the classes were obtained from highest resolution images for all the 12 bands. The classification was then performed on all spatially degraded images of different pixel sizes for both optical (TM) images and radar (SIR-C) images separately by using all six bands. These are shown in figures 7-9. In classification of the spatially degraded images, the statistics of the highest resolution image, i.e. the image with pixel size of 1 , were used as the base spectral signature, and the statistics of the degraded images were compared with this base signature in order to classify the images. The classification accuracy was calculated by comparing the classified image with the ground data image, and counting the number of correctly classified pixels in the whole image. The classification accuracy was calculated for both the TM as well as the SIR-C image at different resolutions. It is obvious that the classification accuracy depends strongly upon the number of classes: the more classes, the higher the chance of misclassification, and thus the lower the information content. However, this merely confirms our assertion that the information content is dependent on the end application, which in this case is the classification into the desired number of classes.

The information content model described in equation (2) was applied to the real data. The same procedure was followed where the values of $k$ and $n$ were calculated by curve fitting. Table 5 shows the $k$ and $n$ values for both sensor systems. In the simulation results, the information content was calculated with respect to the size of the target, i.e. all the 100 pixels were used for the $10 \times 10$ target image. Since the classification accuracy in the analysis of the real data was calculated on a per-pixel basis, the parameter $R$ was taken to be equal to 1 . The curves plotted for both are

Table 4. Six different classes identified in the image data.

\begin{tabular}{ll}
\hline Class & \multicolumn{1}{c}{ Type } \\
\hline Class 1 & Soil \\
Class 2 & Forest \\
Class 3 & Water bodies \\
Class 4 & Urban and highways \\
Class 5 & Pasture \\
Class 6 & Rangeland \\
\hline
\end{tabular}




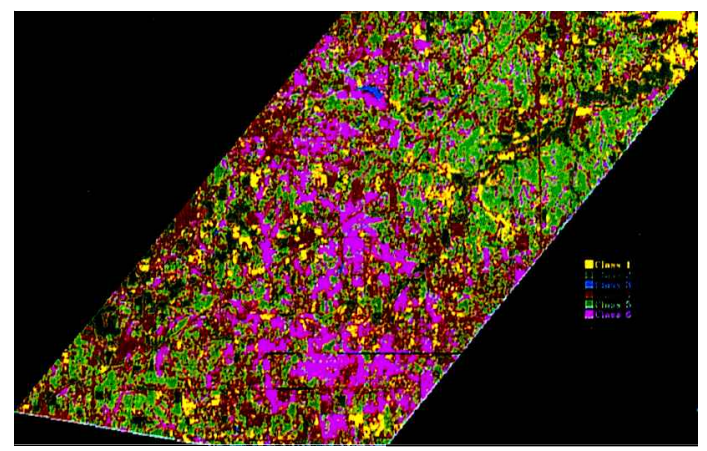

Figure 6. Ground data image obtained after classification using both TM and SIR-C images (12 bands).

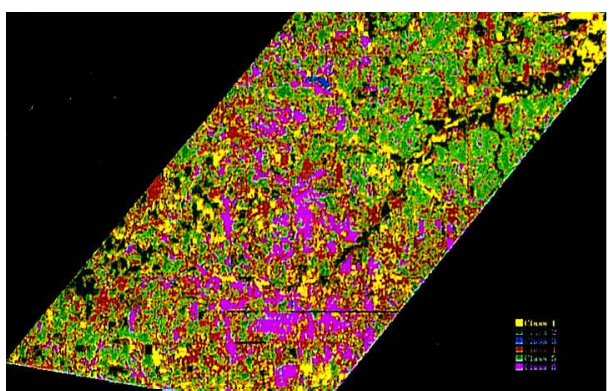

(a)

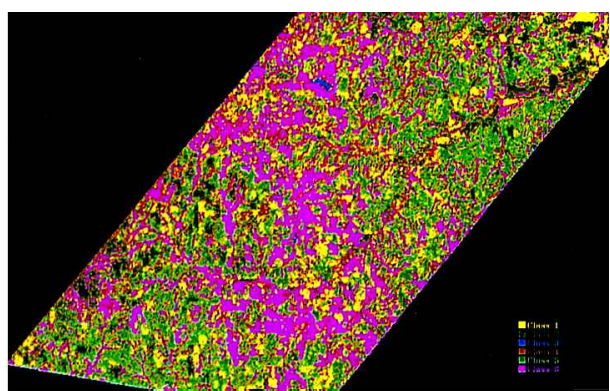

(b)

Figure 7. Classification images for TM and SIR-C at pixel size $=1$. (a) TM classification image, (b) SIR-C classification image.

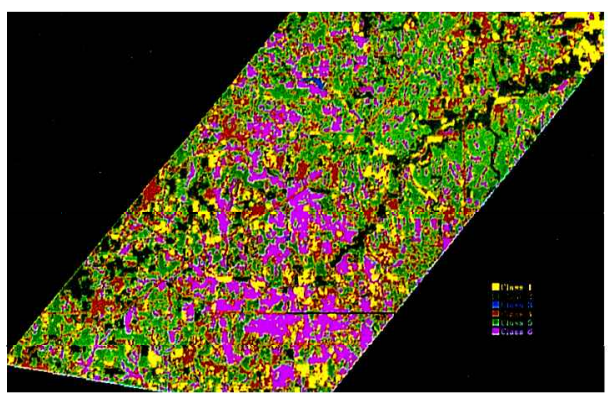

(a)

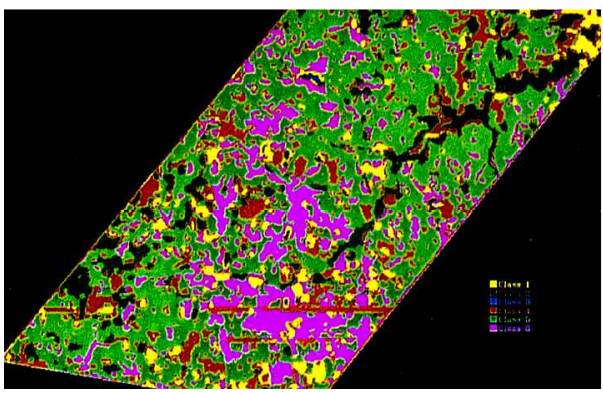

(c)

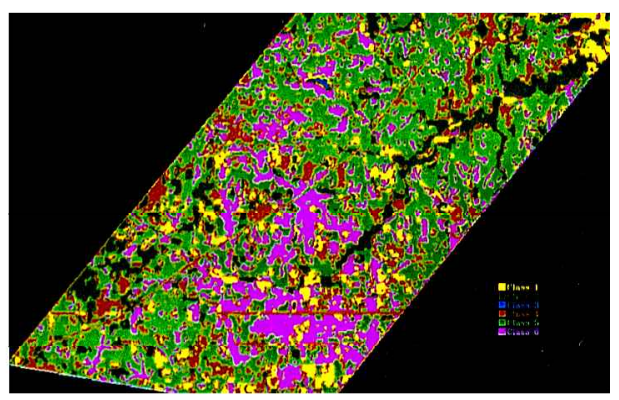

(b)

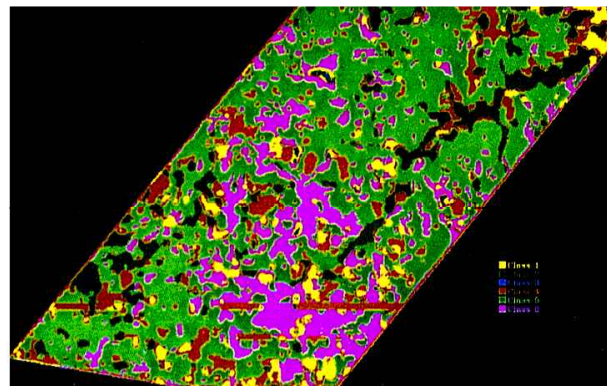

(d)

Figure 8. Effect of varying the pixel size on classification for TM image. Pixel size $=(a) 3$, (b) 7, (c) 11 and $(d) 15$. 


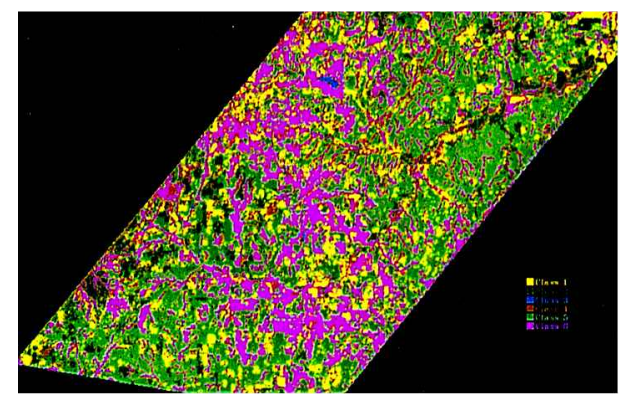

(a)

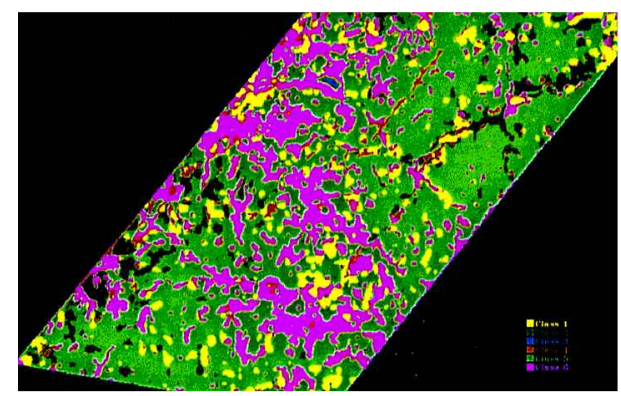

(c)

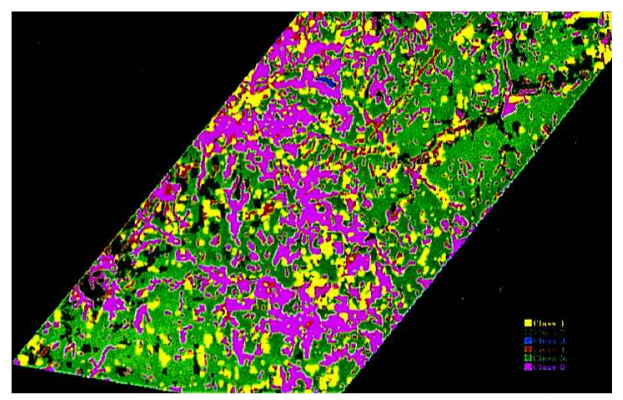

(b)

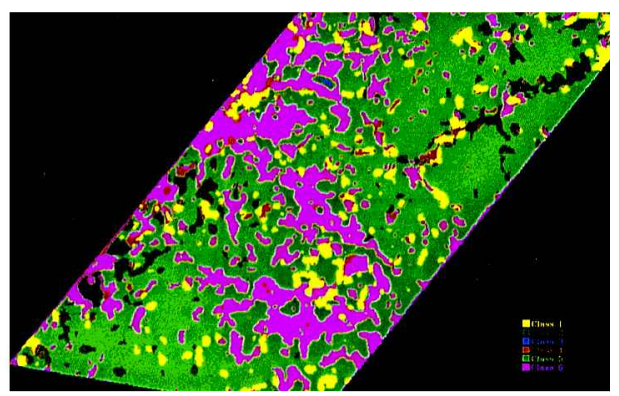

(d)

Figure 9. Effect of varying the pixel size on classification for SIR-C image. Pixel size $=(a) 3$, (b) 7, (c) 11 and (d) 15 .

Table 5. Best-fit model parameter values for real data.

\begin{tabular}{lcc}
\hline Sensor type & $k$ & $n$ \\
\hline TM & 0.1127 & 0.3856 \\
SIR-C & 0.2996 & 0.0776 \\
\hline
\end{tabular}

shown in figure 10. The data points denote the actual classification accuracy, while the solid line is the curve from equation (2) using the values of $k$ and $n$ from table 5 . From the plots, it follows that our exponential information content model is indeed applicable to TM and SIR-C data, and yields an appropriate relation between the information content and the spatial resolution.

At this juncture, it is instructive to discuss the significance of the $k$ and $n$ parameters. The value of $k$ denotes the maximum attainable information content of the system at the best possible spatial resolution, i.e. at $\Delta R=1$, which can be considered to be the basleine information content for the sensor. Although, $I=1$ at $\Delta R=0$, this is a purely hypothetical case. At $\Delta R=1, I=\mathrm{e}^{-k}$; thus, the higher the value of $k$, the lower the baseline information content provided by the sensor. In the above example, the baseline information content for the TM sensor is 0.893 , while for the SIR-C sensor it is 0.741 . Figure 11(a) shows a plot of the information content as a function of pixel size for different values of $k$, while holding $n$ constant at 1 . On the other hand, the parameter $n$ tells us how quickly the information is lost on degrading the spatial resolution for a given $k$. The higher the value of $n$, the faster is the drop or loss in information content as the pixel size increases. This relationship 


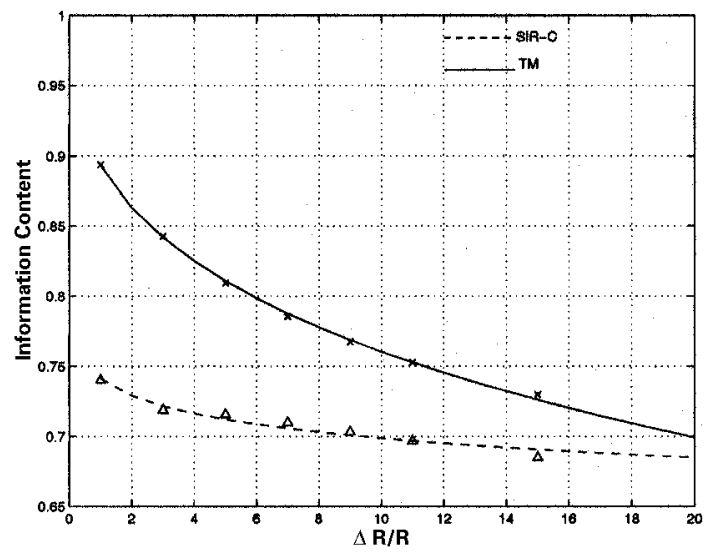

Figure 10. Plots for the model derived results and data points for TM and SIR-C sensors.

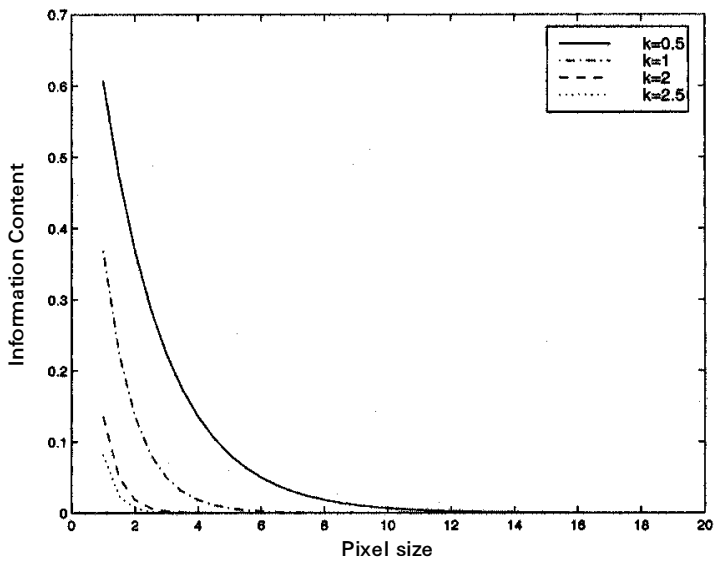

(a)

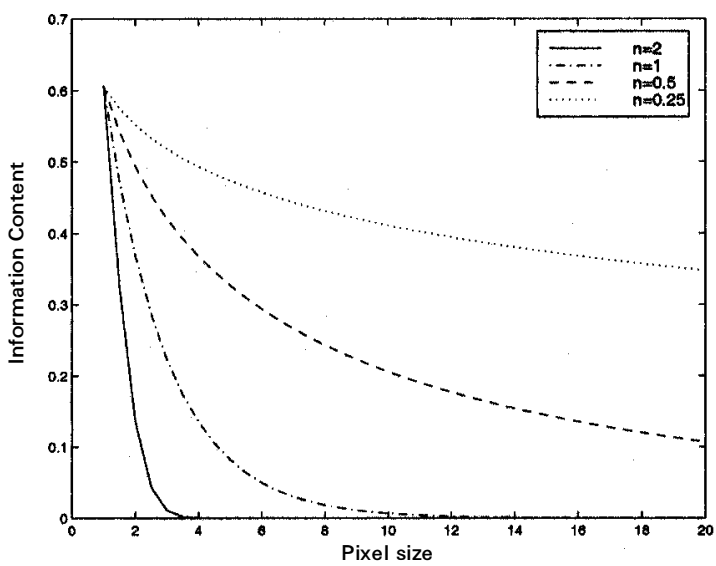

(b)

Figure 11. Plots for information content as function of pixel size with varying values of $k$ and $n$. (a) Varying value of $k, n=1$. (b) Varying value of $n, k=0.5$. 
is seen clearly in figure $11(b)$, where information content is plotted as a function of pixel size for different values of $n$, while holding $k$ constant at 0.5 . In our example, we note that the TM sensor loses information more rapidly than the SIR-C sensor, since it has a higher value of $n$.

Another intersting fact can be deduced from the $k$ and $n$ values of the TM and SIR-C sensors. By equating the information content of both sensors, we can determine the pixel size at which the plots intersect by setting

$$
\exp \left\{-k_{\mathrm{TM}}\left(\frac{\Delta R}{R}\right)^{n_{\mathrm{TM}}}\right\}=\exp \left\{-k_{\text {SIR-C }}\left(\frac{\Delta R}{R}\right)^{n_{\mathrm{SIR}-\mathrm{C}}}\right\}
$$

This occurs at $\Delta R / R=23.9 \approx 24$ (rounded to next highest integer). This tells us that at a pixel size of $720 \mathrm{~m}(24 \times 30 \mathrm{~m})$, SIR-C has the same classification accuracy as that of the TM sensor. This is verified by comparing the information content at a pixel size of 24 for both TM and SIR-C data, which is 0.68 . The information content value for both the sensors are equal, as expected. The classified images at a pixel size of 24 are shown in figure 12. From the above analysis, we conclude that this approach can be used to perform trade-off analysis between sensor systems at different resolutions.

\section{Conclusions}

The study described in this paper was concerned with the quantification and characterization of information content in remote sensing images from various

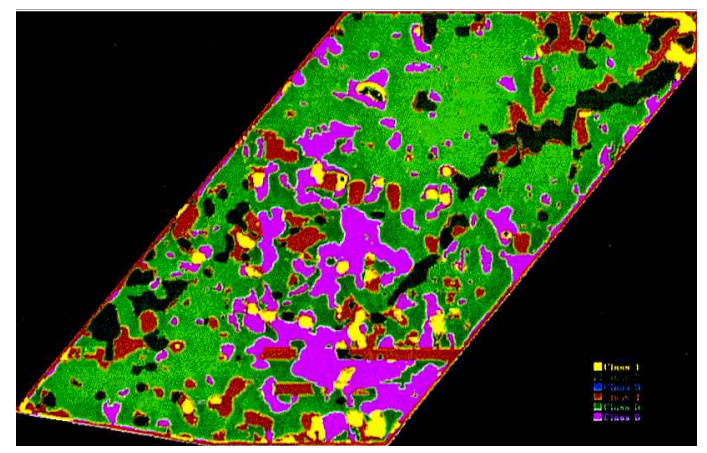

(a)

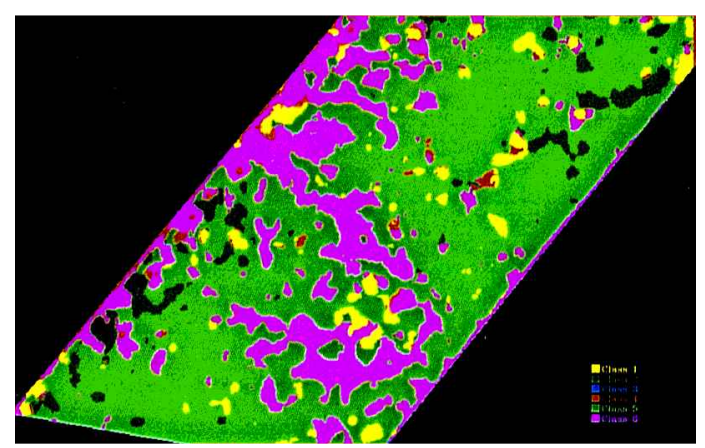

(b)

Figure 12. Classified images at pixel size $=24$. (a) TM. (b) SIR-G. 
sensors. This work is also an attempt to establish the validity of this approach to real sensors, such as the SIR-C/X-SAR and Landsat TM images. The information content formulation using two different approaches, namely image statistics and classification accuracy, were presented and analysed.

Our study shows that information content of an image is related to texture and scene parameters, and it is possible to quantify this value based on the above parameters and the end application of the image analysis. The technique can be used to obtain the optimum resolution needed to yield a particular information content. In the case of multitarget scenes, the classification accuracy, used as a measure of information content, fitted our empirical model quite well. Hence, this model is useful for obtaining the optimum resolution for a particular accuracy for different sensors in both the optical and microwave spectral regimes. However, it must be borne in mind that the information content thus derived is a function of the number of classes.

\section{Acknowledgments}

This work was supported by a grant from NASA under the EPSCoR program, awarded through the Nebraska Space Grant Consortium.

\section{References}

Atkinson, P. M., and Curran, P. J., 1997, Choosing an appropriate spatial resolution for remote sensing investigations. Photogrammetric Engineering and Remote Sensing, 63, $1345-1351$.

BiAn, I., and ButLer, R., 1999, Comparing effects of aggregation methods on statistical and spatial properties of simulated spatial data. Photogrammetric Engineering and Remote Sensing, 65, 73-84.

Blacknell, D., and Oliver, C. J., 1993, Information content of coherent images. Journal of Physics, 26, 1364-1370.

CaO, C., and Lam, N. S. N., 1997, Understanding the scale and resolution effects in remote sensing and GIS. In Scale in Remote Sensing and GIS, edited by D. A. Quattrochi and M. F. Goodchild (Boca Raton, Florida: Lewis Publishers), pp. 57-72.

Dowman, I. J., and Peacegood, G., 1989, Information content of high resolution satellite imagery. Photogrammetria, 43, 295-310.

Frost, V. S., and Shanmugam, K. S., 1983, The information content of synthetic apertaure radar images of terrain. IEEE Transactions on Aerospace and Electronic Systems, 19, 768-774.

Haralick, R. M., Shanmugam, K. S., and Dinstein, I., 1973, Textural features for image classification. IEEE Transactions on Systems, Man, and Cybernetics, 3, 610-621.

Le Hégarat-Mascle, S., Vidal-Madjar, D., Taconet, O., and Zribi, M., 1997, Application of Shannon information theory to a comparison between L- and C-band SIR-C polarimetric data versus incidence angle. Remote Sensing of Environment, 60, 121-130.

Kalmykov, A. I., Sinitsyn, Y. I., Sytnik, O. V., and Tsymbal, V. N., 1989, Information content of radar remote sensing systems from space. Radiophysics and Quantum Electronics, 32, 779-785.

LEE, J. S., 1980, Digital image enhancement and noise filtering by use of local statistics. IEEE Transactions on Pattern Analysis and Machine Intelligence, 2, 165-168.

LEE, J. S., 1986, Speckle suppression and analysis for synthetic aperture radar images. Optical Engineering, 25, 636-643.

Metternicht, G. I., and ZINCK, J. A., 1998, Evaluating the information content of JERS-1 SAR and Landsat TM data for discrimination of soil erosion features. ISPRS Journal of Photogrammetry and Remote Sensing, 53, 143-153.

Moore, R. K., 1979, Tradeoff between pixture element dimensions and noncoherent averaging in side-looking airborne radar. IEEE Transactions on Aerospace and Electronic Systems, 15, 697-708. 
Narayanan, R. M., Desetty, M. K., and Reichenbach, S. E., 1997, Textural characterisation of imagery in terms of information content. Proceedings of Ground Target Modeling and Validation Conference (GTM\&V'97), Houghton, MI, August, (Calumet, MI: Signature Research, Inc.), 1997, pp. 68-74.

Oliver, C. J., 1991, Information from SAR images. Journal of Physics D: Applied Physics, 24, $1493-1514$.

Popatov, A. A., Galkina, T. V., Orlova, T. I., and Khlyavich, Y. L., 1991, A dispersion method for observing deterministic objects as textural optical and radar images of the Earth's surface. Soviet Journal of Communications Technology and Electronics, 36, 1-7.

PRICE, J. C., 1984, Comparison of the information content of data from the LANDSAT-4 Thematic Mapper and the Multispectral Scanner. IEEE Transactions on Geoscience and Remote Sensing, 22, 272-281.

Schowengerdt, R. A., 1997, Remote Sensing: Models and Methods for Image Processing (San Diego, California: Academic Press).

Shen, H. C., and Srivastava, D., 1996, Texture representation and classification: The feature frequency approach. In Advances in Imaging and Electronic Physics, vol.95, edited by P. W. Hawkes (San Diego, California: Academic Press), pp. 387-407.

Tamura, H., Mori, S., and Yamawaki, T., 1978, Textural features corresponding to visual properties. IEEE Transactions on Systems, Man, and Cybernetics, 8, 460-473.

Tomita, F., and Tsuji, S., 1990, Computer Analysis of Visual Textures (Boston, Massachusetts: Kluwer).

US Department of Agriculture, 1996, Hydrology Data Report Washita '94 (Washington, DC: USDA). 